\title{
ON COMPLEX STRICT AND UNIFORM CONVEXITY
}

\section{J. GLOBEVNIK ${ }^{1}$}

ABSTRACT. Strict and uniform $c$-convexity of complex normed spaces are introduced as a natural generalization of strict and uniform convexity. It is proved that the complex space $L_{1}(S, \sigma, \mu)$ is uniformly $c$-convex. An application to analytic functions is given.

Throughout, the open unit disc in the complex plane is denoted by $\Delta$.

Definition 1. A complex normed space $X$ is called strictly $c$-convex if $x, y \in X,\|x\|=1$ and $\|x+\zeta y\| \leq 1(\zeta \in \Delta)$ implies $y=0$.

Definition 2. A complex normed space $X$ is called uniformly $c$-convex if for every $\epsilon>0$ there exists $\delta>0$ such that $x, y \in X,\|x+\zeta y\| \leq 1(\zeta \epsilon$ $\Delta)$ and $\|y\|>\epsilon$ implies $\|x\|<1-\delta$.

Remark. Clearly uniform c-convexity implies strict c-convexity. It is also clear that we get equivalent definitions by replacing $\Delta$ by the set $\{1$, $-1, i,-i\}$.

Given $\delta \geq 0$, let $\omega_{c}(\delta)$ be the supremum of $\|y\|$ taken over all $x, y \in X$ such that $\|x\|=1$ and $\|x+\zeta y\| \leq 1+\delta(\zeta \in \Delta)$. Then one can prove easily that $X$ is strictly (resp. uniformly) $c$-convex if and only if $\omega_{c}(0)=0$ (resp. $\left.\lim _{\delta \backslash 0} \omega_{c}(\delta)=0\right)$.

It is obvious that strict (resp. uniform) convexity of a complex normed space implies its strict (resp. uniform) c-convexity. On the other hand we show that there exist uniformly $c$-convex spaces with no extreme points on the unit sphere.

Thorp and Whitley (see [6]) have proved that the complex space $L_{1}(S, \sigma, \mu)$ is strictly $c$-convex. Here we generalize this by proving that $L_{1}(S, \sigma, \mu)$ is uniformly c-convex. Namely, we prove

Theorem 1. Let $X$ be the complex space $L_{1}(s, \sigma, \mu)$. Let $\delta>0$ and let $x, y \in X$ satisfy $\|x\|=1,\|x \pm y\| \leq 1+\delta,\|x \pm i y\| \leq 1+\delta$. Then

$$
\|y\| \leq \delta^{1 / 2}\left(4+2(1+2 \delta)^{1 / 2}\right) \text {. }
$$

Received by the editors April 26, 1973 and, in revised form, December 3, 1973. AMS (MOS) subject classifications (1970). Primary 30A96.

Key words and phrases. Analytic functions, maximum modulus theorem, strictly and uniformly convex spaces.

${ }^{1}$ This work was supported by the Boris Kidrič Fund, Ljubljana, Yugoslavia. 
Proof. With no loss of generality we may and do assume that $x(s)$ is finite everywhere on $S$. Denote $P=\{s \in S \mid y(s) \neq 0\}$ and define $b(s)=$ $x(s) / y(s)(s \in P)$. Let $c>0$ be arbitrary. Define $P_{1}=\{s \in P:|h(s)|>c\}$, $P_{2}=\{s \in P:|h(s)| \leq c\}$. Clearly $P_{1}, P_{2}$ are measurable. Since $\|x\|=1$ we have

$$
\int_{P_{1}}|y(s)|=\int_{P_{1}} \frac{|x(s)|}{|b(s)|} \leq \frac{1}{s} \int_{P_{1}}|x(s)| \leq \frac{1}{c}
$$

Further, our assumptions imply that

$$
\int_{S}|x(s)+y(s)|+|x(s)-y(s)|+|x(s)+i y(s)|+|x(s)-i y(s)|-4|x(s)| \leq 4 \delta
$$

(note that the integrand is nonnegative by the triangle inequality) so defining

$$
F(s)=|b(s)+1|+|b(s)-1|+|b(s)+i|+|b(s)-i|-4|b(s)| \quad(s \in P)
$$

we have $\int_{P} F(s)|y(s)| \leq 4 \delta$.

Since $F$ is nonnegative by the triangle inequality it follows that

$$
\int_{P_{2}} F(s)|y(s)| \leq 4 \delta .
$$

Now denote $f(r, \theta)=\left|r e^{i \theta}+i\right|+\left|r e^{i \theta}-i\right|-2 r, r \geq 0, \theta$ real. Then for fixed $r$ the function $\theta \mapsto f(r, \theta)$ is continuous, nonnegative, increasing on $(-\pi / 2,0)$, decreasing on $(0, \pi / 2)$ and satisfying $f(r,-\theta)=f(r, \theta), f(r, \theta+\pi)$ $=f(r, \theta)$ for all $\theta$. Hence

$$
f(r, \theta)+f(r, \theta+\pi / 2) \geq f(r, \pi / 4) \text { for all } \theta .
$$

Since

$$
f(r, \pi / 4)=\left(r^{2}+2^{1 / 2} r+1\right)^{1 / 2}+\left(r^{2}-2^{1 / 2} r+1\right)^{1 / 2}-2 r,
$$

it follows that $(f(r, \pi / 4)+2 r)^{2} \geq 4 r^{2}+2$, so $f(r, \pi / 4) \geq\left(4 r^{2}+2\right)^{1 / 2}-2 r$, where the term on the right is positive and decreasing in $r$. Since

$$
F(s)=f(|b(s)|, \arg h(s))+f(|b(s)|, \arg h(s)+\pi / 2) \quad(s \in P)
$$

and since $|h(s)| \leq c\left(s \in P_{2}\right)$ it follows that

$$
|F(s)| \geq\left(4|b(s)|^{2}+2\right)^{1 / 2}-2|b(s)| \geq\left(4 c^{2}+2\right)^{1 / 2}-2 c \quad\left(s \in P_{2}\right) .
$$

Now (3) implies

$$
\int_{P_{2}}|y(s)| \leq 2 \delta\left(\left(4 c^{2}+2\right)^{1 / 2}+2 c\right)
$$


which, together with (2), gives

$$
\|y\|=\int_{S}|y(s)|=\int_{P_{1}}+\int_{P_{2}}|y(s)| \leq 1 / c+2 \delta\left(\left(4 c^{2}+2\right)^{1 / 2}+2 c\right),
$$

and (1) follows by taking $c=1 /\left(2 \delta^{1 / 2}\right)$. Q.E.D.

In particular, the complex space $L(0,1)$ is uniformly c-convex but its unit sphere has no extreme points.

Applications. Let $f$ be a (weakly) analytic function from $\Delta$ to a complex normed space $X$ satisfying $\|f(\zeta)\| \leq 1(\zeta \in \Delta)$. Thorp and Whitley (see [6]) have shown that the implication $\|f(0)\|=1 \Rightarrow f$ is a constant is true in general (which is called the strong maximum modulus theorem) if and only if $X$ is strictly $c$-convex. Here we prove that given $\zeta \in \Delta-\{0\}$, the implication $\|f(0)\|$ is close to $1 \Rightarrow f(\zeta)$ is close to $f(0)$ is true in general if and only if $X$ is uniformly $c$-convex. The only if part follows from the definition of uniform $c$-convexity while the if part follows from

Theorem 2. Let $X$ be a complex normed space and let $f: \Delta \rightarrow X$ be a (weakly) analytic function, satisfying $\|f(\zeta)\| \leq 1(\zeta \in \Delta)$. Then

$$
\|f(\zeta)-f(0)\| \leq(2|\zeta| /(1-|\zeta|)) \omega_{c}(1-\|f(0)\|) \quad(\zeta \in \Delta) .
$$

Proof. If $u$ is a bounded linear functional on $X$ of norm 1 then $|u(f(\zeta))|$ $\leq 1(\zeta \in \Delta)$. Since $\zeta \mapsto u(f(\zeta))$ is analytic on $\Delta$ it follows by the lemma of Harris (see $[1$, p. 1015]) that

$$
|u(f(0))|+(1-|\zeta|)|u(f(\zeta))-u(f(0))| / 2|\zeta| \leq 1 \quad(\zeta \in \Delta-\{0\}) .
$$

Let $\delta=1-\|f(0)\|$ and let $w$ be a unit vector in $X$ with $\|w-f(0)\|=\delta$. Then

$$
\left\|w+\lambda(1-|\zeta|)\left(f\left(\zeta_{o}\right)-f(0)\right) / 2|\zeta|\right\| \leq 1+\delta \quad(\zeta \in \Delta-\{0\}, \lambda \in \Delta)
$$

which, by definition of $\omega_{c}$ implies (4). Q.E.D.

If $X$ is uniformly $c$-convex then $\lim _{\delta \backslash 0} \omega_{c}(\delta)=0$ so (4) implies that $\|f(0)\|$ being close to $1, f(\zeta)$ is close to $f(0)$, uniformly on closed subdiscs. Note that Theorem 2 still holds if $\Delta$ is replaced by the open unit ball of arbitrary complex normed space and the weak analyticity by the weak $G$ analyticity, respectively.

Note added on revision. The author is indebted to Professor L. A. Harris who suggested using $\omega_{c}$ as the measure of (complex) rotundity of the unit ball. After the first version of this paper had been submitted for publication, Professor Harris kindly informed the author that the above two theorems were proved by him in 1971 , even presented in a course but never submitted for publication. 


\section{REFERENCES}

1. L. A. Harris, Schwarz's lemma in normed linear spaces, Proc. Nat. Acad. Sci. U.S.A. 62 (1969), 1014-1017. MR 43 \#936.

2. - A continuous form of Schwarz's lemma in normed linear spaces, $\mathrm{Pa-}$ cific J. Math. 38 (1971), 635-639. MR $46 \# 4167$.

3. E. Hille and R. S. Phillips, Functional analysis and semi-groups, Amer. Math. Soc. Colloq. Publ., vol. 31, Amer. Math. Soc., Providence, R.I., 1957. MR 19, 664.

4. G. Köthe, Topologische lineare Räume. I, 2nd ed., Die Grundlehren der math. Wissenschaften, Band 107, Springer-Verlag, Berlin, 1966; English transl., Die Grundlehren der math. Wissenschaften, Band 159, Springer-Verlag, New York, 1969. MR 33 \#3069; 40 \#1750.

5. D. Pizanelli, Applications analytiques en dimension infinie, Bull. Sci. Math. 96 (1972), 181-191.

6. E. Thorp and R. Whitley, The strong maximum modulus theorem for analytic functions into a Banach space,. Proc. Amer. Math. Soc. 18 (1967), 640-646. MR 35 \#5643.

DEPARTMENT OF MATHEMATICS, UNIVERSITY OF KENTUCKY, LEXINGTON, KENTUCKY 40506

INSTITUTE OF MATHEMATICS, PHYSICS AND MECH ANICS, UNIVERSITY OF LJUBLJ ANA, LJUBLJ ANA, YUGOSL AVIA (Current address) 